\title{
When Paranoia Comes with the Treatment: Psychosis Associated with Tacrolimus Use
}

\author{
João Machado Nogueira ${ }^{a} \quad$ Maria João Freire ${ }^{a}$ Vanessa Vila Nova ${ }^{a}$ \\ Gustavo Jesus b, c \\ aDepartment of Psychiatry and Mental Health, Centro Hospitalar de Setúbal, Setúbal, \\ Portugal; bepartment of Liaison Psychiatry, Centro Hospitalar de Lisboa Central, Lisboa, \\ Portugal; ' Faculdade de Medicina de Lisboa, University Clinic of Psychiatry and Medical \\ Psychology, Lisboa, Portugal
}

\section{Keywords}

Psychosis · Tacrolimus · Neurotoxic · Neuropsychiatric side effects

\begin{abstract}
Tacrolimus is an immunosuppressive drug frequently used in solid organ transplant recipients. This drug has well-documented neuropsychiatric side effects in the literature, although emergence of psychotic symptoms is rare, being only described in a very few case reports. We present a case of a renal transplant recipient with no prior psychiatric history, who developed a severe psychosis secondary to supratherapeutic tacrolimus' blood concentrations. This case highlights the importance of clinical awareness to rare but severe neuropsychiatric effects due to tacrolimus use.
\end{abstract}

\section{Introduction}

Tacrolimus is an immunosuppressive drug, mainly used after solid organ transplant, to reduce the activity of the recipients' immune system, and consequently the risk of organ rejection [1]. Regarding chemical structure, it is a macrolide that reduces peptidyl-prolyl isomerase activity by binding to the immunophilin FKBP-12 (FK506 binding protein) creating a new complex that inhibits calcineurin, impeding T-lymphocyte signal transduction and IL-2 transcription [2-4]. Tacrolimus is structurally and functionally very similar to cyclosporine,

João Machado Nogueira and Maria João Freire contributed equally to this work. 
both calcineurin inhibitors, but tacrolimus can exert its biological properties' at 100-fold lower blood concentrations [2]. It is almost completely metabolised in the liver an intestinal wall, primarily by CYP3A isoenzymes. Therefore, most of its pharmacokinetic variability between subjects is explained by polymorphisms in these isoenzymes' genes. Some other factors that can influence these variability, as well as justify whole blood concentration changes over time, are hepatic dysfunction (by decreasing tacrolimus' clearance), haematocrit, and albumin altered concentrations and corticosteroid dosage [3].

Tacrolimus has been associated with many forms of nephrotoxicity [5] and neurotoxic adverse reactions. In fact, $40-60 \%$ of patients on tacrolimus develop mild-to-moderate neuropsychiatric complaints, such as tremor (one of the first and most common neurologic symptoms is a characteristically fine tremor of the upper extremities), peripheral neuropathy, and anxiety $[4,6]$. Around $5 \%$ of patients treated with tacrolimus develop severe neurotoxic manifestations, including confusion, focal deficits, seizures, posterior reversible encephalopathy syndrome, speech alterations, catatonia, delusions, and hallucinations [7-9].

These side effects have been reported repeatedly after tacrolimus usage in liver and kidney allograph transplantation, but there are only few case reports associating tacrolimus with psychotic episodes [10]. Most cases of neuropsychiatric iatrogenic reactions occur during the first few weeks of tacrolimus administration, but there are reports of severe cases of psychotic episodes occurring many years after therapy initiation. The majority of neuropsychiatric side effects are related to augmented blood concentration $[7,9,10]$ that sometimes arises from drug-drug interactions with frequently used medications, such as antifungal, non-steroidal anti-inflammatory drugs, anticoagulants, or antidiabetics [11].

There are several pathophysiologic mechanisms of tacrolimus-induced neurotoxicity, including vasogenic oedema and increasing nitric oxide production [6-8]. The psychiatric manifestations of severe tacrolimus neurotoxicity (delusional thoughts, hallucinations, disturbed speech, altered mood, or others) might be related to the direct action of calcineurin. Calcineurin is a prevalent protein in the central and peripheral nervous systems and has a direct regulatory effect upon dopaminergic, glutamatergic and GABAergic systems [4, 7], all implicated in the pathophysiology of illnesses with psychotic symptoms, such as schizophrenia or bipolar disorder. Moreover, the calcineurin gene may actually be implicated in the development of schizophrenic symptoms, as this gene and its mRNA have been found to be altered in some patients with schizophrenia either in in vivo and in post-mortem studies [12]. It has also been demonstrated that the administration of antipsychotic drugs diminishes this gene expression but increases the phosphatase activity of calcineurin. These changes seem to be specific to this drug class and mediated by D2/D3 dopamine receptors [12].

\section{Case Report}

We report a case of a rare clinical presentation (psychotic episode) induced by tacrolimus in a renal transplant recipient treated with tacrolimus. Data were collected from interviews with the patient and family members and also review of the available prior clinical records during her admission in the psychiatric ward of Centro Hospitalar de Setúbal, in November of 2019.

A comprehensive search was conducted on the main medical databases including PubMed and Web-of-Science databases (all databases including KCI - Korean Journal Database, MEDLINE, Russian Science Citation Index and SciELO Citation Index), using the MeSH terms: "(psychosis OR delusions OR hallucinations OR side-effects OR psychiatric symptoms OR neurotoxicity) AND (tacrolimus OR calcineurin inhibitors)."

Articles were included in the search regardless of the year of publication or language in which the article was written. The existing literature on the drug was reviewed, including

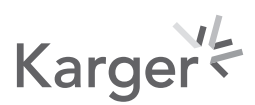


safety profile, pharmacodynamic, and mechanism of action in order to evaluate the link with the patient's clinical presentation.

Inclusion criteria were individuals over 18 years of age, diagnosis of psychosis or psychotic symptoms, and on tacrolimus prescription. The authors independently selected the studies according to the criteria described above. The authors did not assess the risk of bias due to the lack of randomized trials on the theme; the existing literature is of low evidence quality, consisting essentially of case reports and a few series of cases.

Miss S.G.R., 43, single, with medical personal background of chronic renal disease caused by lithiasic chronic pielonefritis and no personal or family history of psychiatric disease. She was kept on haemodialysis between 2014 and 2019 and underwent kidney transplant in December of 2016, in Hospital de Santa Maria. Immunosuppressive therapeutic was initiated with tacrolimus $6 \mathrm{mg}$ twice per day, prednisolone $5 \mathrm{mg} /$ day, and mofetil mycophenolate $1 \mathrm{~g} /$ day, and after a few dose adjustments, the patient was kept on tacrolimus $3 \mathrm{mg}$, mofetil mycophenolate $1 \mathrm{~g}$ and prednisolone $5 \mathrm{mg}$ (daily doses), with a good clinical response, remaining stable until June of 2019.

In June of 2019, Miss S.G.R.'s presented at her assistant nephrologists' consultation with oedema of her feet and legs, decreased urine output and creatinine blood levels of $1.07 \mathrm{mg} /$ $\mathrm{dL}$ (6 months before she had creatinine blood level of $0.77 \mathrm{mg} / \mathrm{dL}$, and around this date, she had also tacrolimus' blood concentration of $6.7 \mathrm{ng} / \mathrm{mL}$, with $3 \mathrm{mg} /$ day). To our knowledge, it was not initiated any specific investigation for this finding, nonetheless, there could have been but with records only on paper.

Those clinical changes led to a therapeutic regimen alteration, namely tacrolimus dose increment from 3 to $5 \mathrm{mg}$ per day without any modification of the doses of the other immunosuppressants). One week after the adjustment, the patient started reporting unusual suspicious thoughts, regarding her family and neighbours.

In September of 2019, she attended a subsequent nephrology appointment and reported new complaints of weight loss and anxiety. Two weeks later, she maintained those symptoms and also began presenting a tremor in both her upper limbs. For these reasons, the tacrolimus' blood concentration was measured and found to be far over the therapeutic interval (5-15 ng/mL): $31.5 \mathrm{ng} / \mathrm{mL}$. The daily dose was diminished from $0.5 \mathrm{mg} /$ day to $4 \mathrm{mg} /$ day, and a new appointment and new blood work were scheduled for the end of October. By then, the concentration of tacrolimus was still elevated: $23.5 \mathrm{ng} / \mathrm{mL}$, and the patient appeared even more distressed, presenting emotional lability (mainly depressed mood), terminal insomnia, disorganized thinking and behaviour with self-reference ideas and persecutory delusions. The daily dose of tacrolimus was, again, altered from 4 to $3 \mathrm{mg} /$ day.

Despite the reduction in the dose of the tacrolimus daily dose operated, it was not enough to control the patient's psychiatric symptoms, and 3 weeks later (mid-November), she was admitted to the psychiatry ward of Centro Hospitalar de Setúbal for delusional persecutory ideas, auditory hallucinations, bizarre behaviours, and severe agitation. She underwent organic evaluation, including a normal head CT scan and normal blood work (showing no chemical signs of infection, no ionic dysregulation, normal thyroid function, no signs of alcohol, or other drugs consumption).

After discussion with the nephrology department, it was decided that tacrolimus was essential and could not be suspended. Thus, its dose was once more reduced from 3 to $2 \mathrm{mg} /$ day and the dose of mycophenolate mofetil was increased to $2 \mathrm{mg} /$ day. Her renal function and the blood concentration were kept under monitoring. Furthermore, antipsychotic medication was initiated (paliperidone, slowly titrated until $9 \mathrm{mg} /$ day) and gradual improving of her mental state occurred. By the seventeenth day after her admission, she did not present psychotic symptoms anymore and started to show partial insight into her clinical condition. She was discharged after 21 days, completely asymptomatic and medicated with paliper-

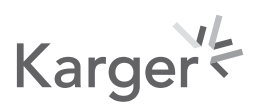


idone $9 \mathrm{mg} /$ day and was referred to the liaison psychiatry consultation and to her assistant nephrologist.

Ten days after discharge, she attended a nephrology consultation. Her renal function was normal and the tacrolimus' blood concentration was within therapeutic levels: $8.9 \mathrm{ng} / \mathrm{mL}$ (on $2 \mathrm{mg}$ /day). Later on, paliperidone was switched to olanzapine $7.5 \mathrm{mg}$ at night due to the development of extrapyramidal side effects. Since then, she has been attending both nephrology and psychiatry consultations and was kept on the referred psychiatric medication, with clinical stability and complete remission of psychotic symptoms. Later on, olanzapine was suspended and as there was no symptomatic relapse, the patient was discharged at the last consultation of psychiatry.

\section{Discussion/Conclusions}

The vast majority of primary and potentially severe mental illnesses start in the second or third decades of life. Therefore, the relatively sudden appearance of psychotic symptoms with an atypical clinical presentation (sudden onset, poorly systematized delusional ideas in a middle-aged patient, with no previous psychiatric history), raises the suspicion of possible secondary causes.

We presented a clinical case of a woman whose emergence of psychiatric symptoms was concomitant with the increase of the tacrolimus daily dosage, leading to its toxic levels of blood concentration.

Even though the majority of neuropsychiatric complications associated with tacrolimus use arise during the first weeks of treatment, there are also cases, like this one, that only appear after years of tolerability.

On a retrospective analysis of the case, we can hypothesize that by June of 2019 the patient was already showing mild neuropsychiatric symptoms that were not interpreted as such by her assistant nephrologist, and therefore not addressed. Consequently, those symptoms worsened in the following months, ultimately resulting in hospital admission in a psychiatric ward.

During the hospital stay, other secondary causes to the psychotic outbreak were excluded and the tacrolimus' blood concentration at toxic levels shortly before her admission was the only pathological organic element identified, making this the most certain causal factor for the psychotic outbreak.

Tacrolimus daily dose was gradually reduced until therapeutic blood levels were obtained and antipsychotic medication was initiated. There was a relatively rapid recovery, with complete remission of symptoms and gain of insight by the patient, who is currently medically stable and without psychiatric symptomology.

The patient's absence of previous psychiatric history, the atypical clinical presentation, the emergence of psychotic symptoms concomitantly with tacrolimus' toxic blood concentration and the clinical resolute with the normalization of these toxic levels, make it very likely that this was a psychotic episode induced by tacrolimus toxicity.

Although rare, the effects on the central nervous system of tacrolimus can be severe [13]. Calcineurin has a modulatory effect upon glutamatergic pathways (via NMDA receptors) and GABA-mediated responses (by regulation of desensitization of GABA receptors) [4] and is also involved in dopamine signal transduction [14]. These neurotransmitters have knowingly been implicated in the emergency of psychotic symptoms and behaviour abnormalities in schizophrenia and other psychotic disorders, and calcineurin seems to be implicated in the process. There have been studies showing that calcineurin gene is altered in the genome of schizophrenic patients $[12,14]$ and also that the chronic administration of antipsychotics has

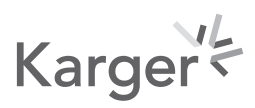


Case Reports in Nephrology and Dialysis

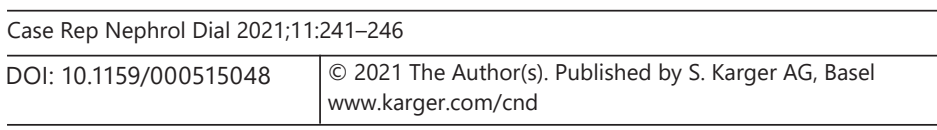

Nogueira et al.: Tacrolimus-Induced Psychosis

an effect of the genetic expression of calcineurin [14]. These can be putative mechanisms implicated, as in the case here presented, in the emergency of psychotic outbreaks associated with tacrolimus chronic administration. Therefore, clinicians should be alert to that when managing this drug, avoiding its supratherapeutic blood levels. If neurotoxic symptoms should appear, tacrolimus' dosage should be adjusted or at all discontinued (if possible), to get full clinical resolution, in some cases without the need to use antipsychotics. In the future, regarding the management of these patients, there is a tendency that personalized drug therapy, with novel methods for drug monitoring, will better predict the dose-concentrationresponse relationship. Also, a close serum level monitoring might contribute to prevent neurotoxicity associated with immunosuppressants, and ultimately improve patient care [9].

This case enhances the importance of effective collaboration between medical specialities, showing that a multidisciplinary approach is key in this kind of cases. It also warns physicians for a careful investigation of new psychotic symptoms of sudden onset, before attributing those to a primary psychiatric disease, highlighting the importance of a timely psychiatric evaluation to take into account the possibility of a secondary organic disorder.

\section{Statement of Ethics}

Patient signed a written consent authorizing publication, in compliance with the Helsinki Declaration. No identifiable data from the patient was disclosed in the text.

\section{Conflict of Interest Statement}

The authors declare that they have no conflicts of interest.

\section{Funding Sources}

The authors declare that they have not conflicts of interest and do not receive any funding.

\section{Author Contributions}

All the authors had fulfilled the authorship criteria and the manuscript is in accordance with the instructions for the authors and with the ethical and legal principles (according to Declaration of Helsinki by the World Medical Association). J.N. and M.F. contributed to the conception, design, and writing of the manuscript and final approval of the version to be published. G.J. contributed by content review, and questions related to the accuracy and integrity of any part of the work were appropriately investigated and resolved.

\section{References}

1 Manvizhi S, Mathew BS, Fleming DH, Basu G, John GT. Combined approach with therapeutic drug monitoring and pharmacogenomics in renal transplant recipients. Indian J Nephrol. 2013;23(23):71-3.

2 Liu J, Farmer JD, Lane WS, Friedman J, Weissman I, Schreiber SL, et al. Calcineurin is a common target of cyclophilin-cyclosporin A and FKBP-FK506 complexes. Cell. 1991;66(4):807-15.

3 Staatz CE, Tett SE. Clinical pharmacokinetics and pharmacodynamics of tacrolimus in solid organ transplantation. Clin Pharmacokinet. 2004;43(43):623-53. 
4 Bechstein WO. Neurotoxicity of calcineurin inhibitors: impact and clinical management. Transpl Int. 2000; 13(5):313-26.

5 Nayagam LS, Vijayanand B, Balasubramanian S. Massive pleural effusion in a renal transplant recipient on tacrolimus. Indian J Nephrol. 2014;24(5):318-20.

6 Chopra A, Das P, Rai A, Kuppuswamy PS, Li X, Huston J, et al. Catatonia as a manifestation of tacrolimusinduced neurotoxicity in organ transplant patients: a case series. Gen Hosp Psychiatry. 2012;34(34):209. e9-e11.

7 Sikavi D, Mcmahon J, Fromson JA. Catatonia due to tacrolimus toxicity 16 years after renal transplantation: case report and literature review. J Psychiatr Pract. 2019;25(25):481.

8 Wijdicks EFM. Neurotoxicity of immunosuppressive drugs. Liver Transpl. 2001 Nov;7(11):937-42.

9 Wu Q, Marescaux C, Wolff V, Jeung MY, Kessler R, Lauer V, et al. Tacrolimus-associated posterior reversible encephalopathy syndrome after solid organ transplantation. Eur Neurol. 2010;64(64):169-77.

10 Bersani G, Marino P, Valeriani G, Cuoco V, Zitelli C, Melcore C, et al. Manic-like psychosis associated with elevated trough tacrolimus blood concentrations 17 years after kidney transplant. Case Rep Psychiatry. 2013; 2013:926395.

11 Soubhia RMC, Mendes GEF, Mendonça FZ, Baptista MAS, Cipullo JP, Burdmann EA. Tacrolimus and nonsteroidal anti-inflammatory drugs: an association to be avoided. Am J Nephrol. 2005;25(4):327-34.

12 Rushlow WJ, Seah C, Sutton P, Bjelica A, Rajakumar N. Antipsychotics affect multiple calcium calmodulin dependent proteins. Neuroscience. 2009;161:877-86.

13 Veroux P, Veroux M, Pulliatti C, Valvl M, Macarone M, Cappello D. Severe neurotoxicity in tacrolimus-treated living kidney transplantation in two cases. Urol Int. 2003;71(4):433-4.

14 Rushlow WJ, Seah YH, Belliveau DJ, Rajakumar N. Changes in calcineurin expression induced in the rat brain by the administration of antipsychotics. J Neurochem. 2005;94(94):587-96. 